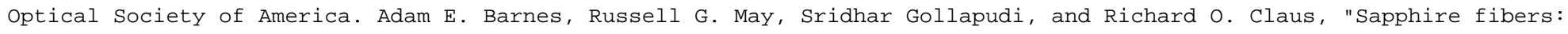
optical attenuation and splicing techniques," Appl. Opt. 34, 6855-6858 (1995). doi: 10.1364/AO.34.006855

\title{
Sapphire fibers: optical attenuation and splicing techniques
}

\author{
Adam E. Barnes, Russell G. May, Sridhar Gollapudi, and Richard O. Claus
}

\begin{abstract}
The optical attenuation in sapphire fibers was examined. Attenuation was found to depend heavily on injection conditions. A number of techniques for making sapphire-silica fiber splices were attempted, with an effort toward optimizing injection conditions in the sapphire fiber. The most successful of these techniques, interior capillary-tube splicing, produced robust splices with an attenuation of less than $1 \mathrm{~dB}$.
\end{abstract}

\section{Introduction}

Sapphire fiber sensors hold great promise for hightemperature-sensing applications because of their small size, their immunity to electromagnetic interference, and their high melting temperature, which exceeds $2000{ }^{\circ} \mathrm{C}$. Unfortunately, sapphire fibers are expensive and have a high optical attenuation, which limits the length of sapphire fiber that can be used. Consequently, a practical sapphire sensor will have a sapphire fiber only in the sensor head and will use a silica fiber for transmitting signals to and from the test region. For realizing such a sensor system, a suitable method for splicing sapphire to silica had to be developed. This was no small task, as sapphire and silica fibers are radically different. Silica fiber is a glassy, isotropic, amorphous solid that makes a weakly guiding waveguide with a core and cladding that have indices of refraction of roughly 1.48 and 1.46 , respectively, with a low $\left(0.53 \mu \mathrm{m} / \mathrm{m} \mathrm{K}^{-1}\right)$ coefficient of thermal expansion (CTE) and a softening temperature of 800 to $1000{ }^{\circ} \mathrm{C}$ (depending on the exact dopants used). Sapphire, on the other hand, is a single anisotropic crystal that makes a strongly guiding waveguide that consists of only a core with a refractive index of 1.76 (approximately, as sapphire is birefringent), a high CTE $\left(8.4 \mu \mathrm{m} / \mathrm{m} \mathrm{K}^{-1}\right)$, and a melting temperature of over $2000^{\circ} \mathrm{C}$. Unlike silica fiber and bulk sapphire, which have undergone exten-

The authors are with the Bradley Department of Electrical Engineering, Fiber and Electro-Optic Research Center, Virginia Polytechnic Institute and State University, Blacksburg, Virginia 24060-0111.

Received 23 December 1994; revised manuscript received 21 April 1995.

0003-6935/95/306855-04\$06.00/0.

(C) 1995 Optical Society of America. sive study, the optical properties of the sapphire fiber are not well known, and so the optical characterization of the sapphire fiber was the first step in producing a practical sapphire-silica splice. Subsequently many splicing methods were examined, including diffusion-enhanced splicing, splicing with coated fibers, and splicing inside of capillary tubes. The last of these methods demonstrated the most promise by producing simple, mechanically strong splices with losses of less than $1 \mathrm{~dB} .^{1}$

\section{Optical Characterization}

The initial optical characterization of the sapphire fiber concentrated on the analysis of optical attenuation under various conditions. Optical attenuation was measured with the cutback method. For this procedure, we first prepare the sapphire fiber by polishing both of its endfaces. Because the sapphire fiber is grown as a single anisotropic crystal, it cannot be cleaved in a manner similar to that used for glass fibers. The sapphire fiber is injected with light at one end, and the output is measured at the other end with a photodetector. The length of the fiber and the optical output are recorded. The fiber is then cut back to a short length, and the broken endface is repolished. The cutback segment of the fiber is then again injected with light. The logarithm of the ratio of the two optical output measurements divided by the difference in fiber length gives the attenuation, in decibels per unit length in the sapphire fiber, that is,

Attenuation (dB/length)

$$
=\frac{1}{(\Delta L)} 10 \log _{10} \frac{\text { Cut-back measurement }}{\text { Initial measurement }},
$$

where $\Delta L$ is the difference in length between the initial and the cutback lengths. Note that, for the 
experiments detailed here, the optical output measurements were adjusted for noise and input power levels. Also, the optical properties of bulk sapphire indicate that sapphire has a very nearly uniform optical transmission of between 800 and $1300 \mathrm{~nm}$, so that, although all testing in these experiments was performed at $830 \mathrm{~nm}$, the results can be generalized to other wavelengths. The attenuation measurement station that was constructed used an 830-nm pigtailed laser diode as the light source. The optical output was measured by a large surface area photodetector with variable gain resistor circuitry connected to an oscilloscope. Three different injection conditions were tested, each with a different numerical aperture (NA). The largest NA used a multimode fiber, rated to have a $0.25 \mathrm{NA}$. To ensure that the modes in the multimode fiber were evenly excited, the single-mode pigtail was connected to a mode scrambler before it was coupled to the multimode fiber, as shown in Fig. 1. The mode scrambler consisted of three 1-m-long pieces of silica fiber concatenated in series by fusion splices. The fibers were $5 / 125-\mu \mathrm{m}$

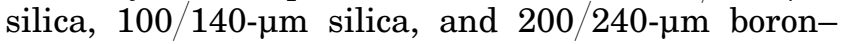
silica fibers; the ratio represents the core diameter to cladding diameter ratio. The second injection condition used just the single-mode pigtail from the laser diode with a NA rated at 0.11 . The smallest NA of 0.09 was achieved with a series of lenses and pinholes to focus the light into the sapphire fiber, as shown in Fig. 2. It was found that the lower the NA of the injecting light source, the lower the attenuation in the fiber. It is hypothesized that this is because the higher NA's excite higher-order modes, which are attenuated strongly, so that a significant fraction of the light power is lost. Smaller NA's tend to excite only lower-order modes, which are not attenuated as heavily, resulting in ostensibly lower optical losses.

Because of the modal sensitivity of the sapphire fiber, injection conditions are critical in any optical measurement of the fiber. In addition to the NA, other important parameters include the condition of the fiber endfaces on both the sapphire fiber and the injecting silica fiber, the modal pattern in the injecting fiber, and any curves or bends in the sapphire fiber. Although the fiber endfaces can be polished to an smooth finish $(0.3 \mu \mathrm{m})$, it is difficult to achieve a polish that is exactly perpendicular to the axis of the fiber. This essentially results in an angular misalignment between the sapphire fiber and the injecting fiber. In addition to coupling losses normally associated with angular misalignment, there is further attenuation in the sapphire fiber, in this case because the angle of injected light rays is altered, which excites higher-order modes in the sapphire and so

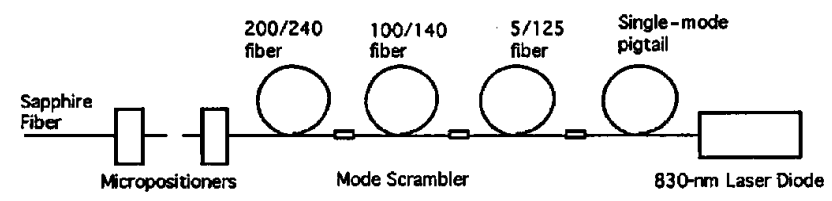

Fig. 1. Injection of a sapphire fiber by a mode scrambler.

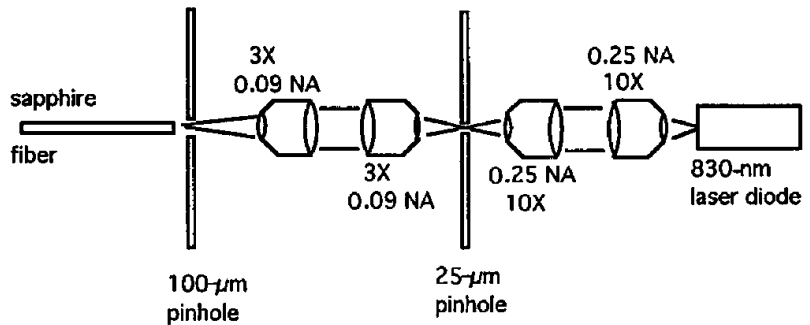

Fig. 2. Lens-pinhole configuration for restricted NA injection.

results in greater attenuation. The modal pattern in the injection fiber is also of importance because it directly affects the modal pattern in the sapphire fiber. Different modal patterns in the injection fiber will excite higher- or lower-order modes in the sapphire, which will result in different attenuation measurements. Care must also be taken to keep the sapphire fiber as straight as possible, because bends in the sapphire tend to couple lower-order modes into higher-order modes. Hence any curve in the fiber will result in greater attenuation.

Different injection conditions can result in attenuation measurements that vary by as much as 2 orders of magnitude, with losses of typically between 2 and $0.02 \mathrm{~dB} / \mathrm{cm}$, depending on the injection conditions and the quality of the fiber. The lowest attenuation measured was $0.016 \mathrm{~dB} / \mathrm{cm}$ in a $100-\mu m$-diameter optical grade fiber grown at the University of Southern Florida by the use of the lens-pinhole injection configuration. The highest attenuation in these experiments was $2.16 \mathrm{~dB} / \mathrm{cm}$, which was measured in a 165- $\mu$ m-diameter structural grade fiber from Saphikon by the use of multimode fiber injection. Because optical transmission through sapphire fiber is so potentially variable, relevant attenuation measurements actually depend on the specific sapphire fiber application.

\section{Sapphire-Silica Splices}

The actual splicing of the silica fibers proved challenging because the physical disparities between the fibers adversely affected the mechanical strength of the splices. Additionally, normal splicing difficulties of alignment and reflections at the interface were exacerbated by the modal sensitivity and the high refractive index of the sapphire. The methods attempted were diffusion-enhanced splices, splices with silica- or glass-coated fibers, and splices with capillary tubing.

The diffusion-enhanced splice was initially fabricated by the use of sapphire and silica rods, which were easier to handle. We made the splice by aligning the sapphire and the silica endfaces with micropositioners and heating them gradually with an oxypropane torch to promote diffusion between the interface. This procedure was subsequently performed with fibers, but the heating in this instance was done in a small, ceramic oven. Although scanning electron micrographs did indicate that diffusion may have occurred, there was also a formation of mullite crys- 
tals (an intermediate mixture of silica and alumina) at the interface. These crystals were not conducive to good optical transmission and made the splices quite fragile. ${ }^{1}$

Several sapphire-silica splices were made with one or both of the fibers coated with fused silica or aluminosilicate glass (ALG) and then spliced on an arc fusion splicer. Initially, uncoated sapphire and silica were attempted, and although the resulting splices were somewhat stronger than the diffusion-enhanced splices, there was still evidence of mullite crystal formation, which resulted in unacceptably high optical losses, $\sim 10 \mathrm{~dB}$. To prevent mullite formation, the sapphire fiber was coated with silica by chemicalvapor deposition. The two fibers were then spliced together on the arc fusion splicer as before. Although this resulted in a strong splice, the optical losses were still of the order of $10 \mathrm{~dB}$, which was likely due to imperfect alignment of the sapphire and silica fibers. Scanning electron micrographs did not reveal evidence of mullite crystal formation. ALG was also used as a coating. ALG has a lower softening point than fused silica does, and its intermediate refractive index between that of silica and sapphire reduces Fresnel reflections in the splice, making it a good choice for a binder. The ALG was melted in a crucible, and the fibers to be coated were dipped into the molten glass. Although coatings were attempted on both the silica and the sapphire, the coated silica fiber tended to crack on cooling, probably because of the different CTE's between the silica and the ALG. Initial attempts achieved splice losses of $\sim 5 \mathrm{~dB}$, although they were not very strong splices. To lower the splice losses, attempts to improve the alignment were made by the injection of light through the fibers to be spliced and the adjustment of the fibers on the arc fusion splicer's micropositioners until maximum light transmission was obtained. This approach was unsuccessful, however, because it was found that the fibers shifted inside the molten ALG as it was heated and cooled. Subsequently, the ALG was polished parallel to the endface of the sapphire fiber, down to a thickness of a few micrometers, to allow better alignment of the fibers. Thinner ALG coatings resulted in lower optical losses but mechanically weaker splices. The lowest loss achieved with this method was $1.5 \mathrm{~dB}$. To strengthen these splices, silica tubes were epoxied in place over the splice, which resulted in satisfactorily robust splices. Although this technique was capable of producing good splices, it was lengthy, involved, and difficult to perform. ${ }^{1}$

To improve the alignment of the fibers, which had up to this point proved to be the most critical parameter for low-loss splices, splicing was attempted inside the capillary tubes that had inner diameters closely matched to the outer diameters of the fibers to be spliced. A short piece of ALG was used as a binder inside the tube, and the whole assembly was spliced together on an arc fusion splicer, as shown in Fig. 3. Initial attempts were made with fused-silica

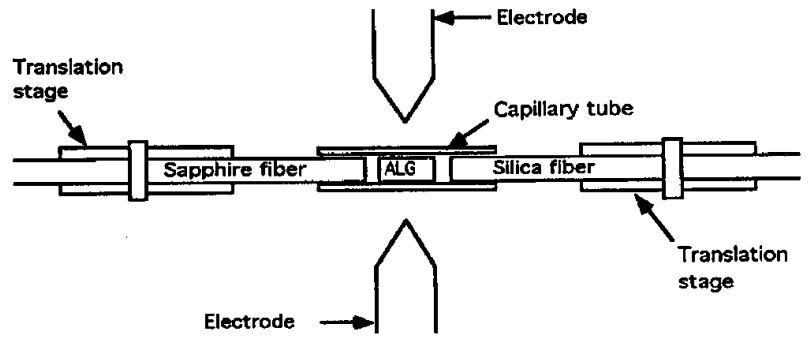

Fig. 3. Setup for capillary-tube splice technique.

tubing. This proved inadequate, as the different CTE's between the tube and the ALG caused the tube to crack. Stainless-steel tubing was also used in this approach. Unfortunately, because the steel tubing was opaque, the splice could not be monitored during the splicing procedure. This resulted in either the fiber endfaces' not being close enough together or in the binder's being overheated, which produced bubbles in the ALG. In either case, the optical qualities of the splice were poor. Finally, a sapphire tube was used, which did not have the problems of either the silica or the steel tubes. The tube was produced by Saphikon with an edge-defined film-fed growth technique. Splices with optical losses consistently below $1 \mathrm{~dB}$ were produced in this fashion. The exposed silica fiber proved to be the weakest part of the splice, so this was encased in a silica tube (see Fig. 4). The final splices were mechanically as strong as the silica fiber itself. The sapphire capillary-tube splices have the additional advantage of being relatively easy to fabricate.

We computed the splice losses noted above by measuring the total loss in the silica-splice-sapphire fiber and then subtracting the optical attenuation measured for the sapphire fiber itself (the silica fiber was assumed to have negligible loss). However, as mentioned above, the sapphire fiber attenuation is very sensitive to injection conditions. Because of mixing of modes in the splice, the injection conditions in the spliced sapphire were different from those during the attenuation measurement. To ascertain whether splice losses were influenced by mode mixing, a 89.15-cm-long section of 100- $\mu$ m-diameter sapphire fiber (with attenuation previously measured to be $0.016 \mathrm{~dB} / \mathrm{cm}$ ) was spliced to a single-mode silica fiber (with the sapphire capillary-tube method), and the splice loss was measured. Then the sapphire was cut back to $3.55 \mathrm{~cm}$, repolished, and the splice loss was remeasured. The splice loss with a short

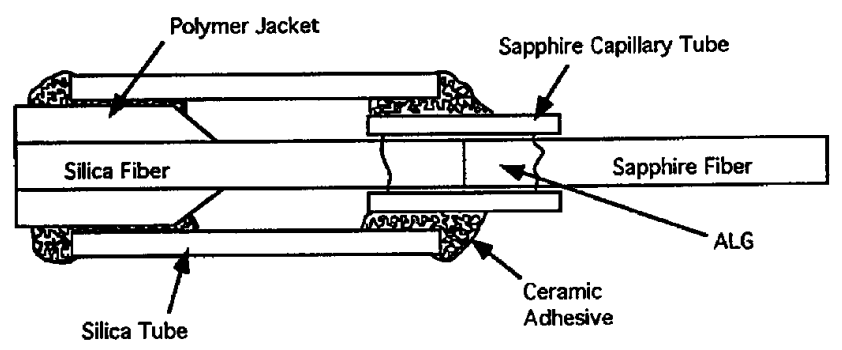

Fig. 4. Finished sapphire capillary-tube splice. 
section of sapphire was measured to be lower than with the long section of sapphire. This would suggest that the splice tends to act as a mode mixer between the silica and the sapphire, exciting higherorder modes in the sapphire and resulting in more loss in the sapphire fiber. Consequently, the splice losses listed above are probably consistently too high, as the sapphire fiber is more lossy when spliced than during the attenuation measurements. Although the measurement techniques used in these experiments can give an idea of the relative optical losses in different splicing techniques, acceptable overall loss should be computed on basis of the parameters needed for a given application, particularly with an eye toward duplicating the injection conditions.

\section{Conclusion}

Although much work remains to be done with sapphire fibers, considerable insight has been gained into the optical properties of sapphire fiber, and splicing efforts have been significantly advanced. Sapphire fiber optical attenuation, although relatively unaf- fected by wavelength in the region of interest, is extremely sensitive to injection conditions because of the strong attenuation of higher-order modes. Because of this, sapphire-silica splices are particularly vulnerable to lateral and angular misalignments, which result in more higher-order modes' being excited in the sapphire fiber. The relatively simple sapphire capillary-tube splice provides excellent fiber alignment, resulting in mechanically robust splices with optical losses under $1 \mathrm{~dB}$.

This work was sponsored by NASA LaRC under the NASA Research Grant NAG-1-1275. Initial splice work was done by Kent Murphy and Shari Feth at the Virginia Polytechnic Institute and State University, with suggestions from David R. Clarke at University of California, Santa Barbara.

\section{Reference}

1. S. Gollapudi, "Sapphire optical fibers: splicing and sensing applications," Master's thesis (Virginia Polytechnic Institute and State University, Blacksburg, Va., 1992). 